\author{
R. Arlegi \\ M. Ballester \\ M. Besada \\ J.R. De Miguel \\ J. Nieto \\ C. Vázquez \\ D.T.2006/09
}




\title{
On the equivalence of the two existing extensions of the leximax criterion to the infinite case
}

\author{
R. Arlegi ${ }^{\mathrm{a}}$, M. Ballester ${ }^{\mathrm{b}}$, M. Besada $^{\mathrm{c}}$, J.R. De Miguel $^{\mathrm{a}}$, \\ J.Nieto $^{\mathrm{a}}$ and C. Vázquez ${ }^{\mathrm{c}}$ \\ ${ }^{\text {a } U n i v e r s i d a d ~ P u ́ b l i c a ~ d e ~ N a v a r r a ~}$ \\ ${ }^{\mathrm{b}}$ Universidad Autónoma de Barcelona \\ ${ }^{\mathrm{c}}$ Universidade de Vigo
}

\begin{abstract}
Using a common framework, we consider the two existing extensions of the leximax criterion to infinite environments (Arlegi et al. (2005) and Ballester and De Miguel (2003)), and show that, though the respective definitions of the rules and their axiomatic characterizations appear to differ considerably, they actually propose the same extension of the leximax criterion to the infinite case.
\end{abstract}

Key words: preferences, utility, leximax

JEL classification: $\quad$ C60

\section{Introduction}

Consider a given set of alternatives $X$ over which there is defined a preference ordering $R$. Consider also the problem of ranking all the possible finite subsets of $X$. This formal problem can be understood in many different decisional contexts. Specifically, if we interpret the subsets as opportunity sets, and take the ranking of these to reflect the degree of freedom of choice with which they provide the decision maker, the mentioned problem is a natural way to

1 Please address correspondence to: Ritxar Arlegi. Department of Economics. Public University of Navarra. Campus Arrosadia. 31600 Pamplona. Navarra. Spain. pho: ++ 948 169365. fax: ++ 34948 169721. e-mail: rarlegi@unavarra.es 
formally describe people's valuation of freedom of choice. This is the focus of many authors in the so-called freedom of choice literature. ${ }^{2}$

Among the many different plausible rules one could imagine, one attractive proposal is the leximax ranking, which is defined, and axiomatically characterized by Bossert et al. (1994). This rule compares any two sets of alternatives by first looking at the best alternative in each set (with respect to $R$ ); if the comparison is not decisive (the elements are indifferent), then the rule considers the second-best alternative in each set, and so the procedure continues, if necessary, until there are no more alternatives to be compared in either or both of the sets. In the first case, the set with the greater number of alternatives is declared to be better; in the second, the two sets are indifferent.

The interest of this rule lies in the fact that it incorporates the intrinsic value of freedom of choice (any enlargement of an opportunity set leads to a strict improvement, unlike the standard indirect utility criterion), and the instrumental value of freedom of choice (preferences over the individual alternatives in the set matter, unlike the purely cardinalist rule proposed by Pattanaik and $\mathrm{Xu}(1990))$

A major drawback of the leximax rule as established in Bossert et al. (1994), however, is that it is not well defined for the case of infinite opportunity sets, and is therefore not applicable in meaningful economic contexts, such as the ranking of standard budget sets, which are typically compact (and infinite).

In an attempt to address this shortcoming, Ballester and De Miguel (2003) and Arlegi et al. (2005) have separately proposed and axiomatically characterized an extension of the leximax rule to the infinite case. The two proposals look very different both in their formulation and their axiomatic structure. To the best of our knowledge, moreover, these are, to date, the only two existing extensions of the leximax rule to the infinite case.

The aim of this study is to prove that, despite their apparent differences, the two extensions of the leximax are equivalent. In Section 2, we introduce the basic notation and definitions. Section 3 presents the definitions of the two extensions of the leximax as originally proposed by their respective authors. Section 4 contains the equivalence result and its proof. In Section 5 we make some comments on the logical relationship between the axioms used for the characterization in each article, and finish with some concluding remarks in Section 6.

2 The interested reader can find a complete survey of this literature in Barbera et al. (2004). 


\section{Notation and definitions ${ }^{3}$}

$\mathbb{N}$ and $\mathbb{R}$ denote the set of all positive integers and the set of all real numbers, respectively, and $\mathbb{R}^{n}$ is the $n$-fold cartesian product of $\mathbb{R}$. Let $X \subset \mathbb{R}^{n}$ be a nonempty set of alternatives. In order to ensure that the axioms used are independent, $X$ is assumed to contain at least three elements.

Let $R$ be a complete, reflexive, transitive ordering on $X$ that can be represented by a utility function. The asymmetric and symmetric parts of $R$ are denoted, respectively, by $P$ and $I$. The set of all subsets of $X$ is denoted by $2^{X}$. For any $A \subseteq X, \mathcal{F}(A)$ denotes the set of the finite subsets of $A$, and $\neg$ denotes the logical negation.

Let $\succeq$ be a preference relation (complete, reflexive and transitive ordering) defined on $2^{X}$. We write $A \succeq B$ to indicate that "set $A$ is at least as good as $B$ ". We define the associated strict preference and indifference relations in the usual way: $\forall A, B \subseteq X, A \succ B$ iff $A \succeq B$ and $\neg(B \succeq A)$, and $\forall A, B \subseteq X$, $A \sim B$ iff $A \succeq B$ and $B \succeq A$.

We assume $A \succ \emptyset$, for all $A \subseteq X, A \neq \emptyset$.

\section{The two extensions of the leximax criterion to the infinite case}

First, a preference relation on $\mathcal{F}(X)$ is considered. We denote by $\succeq_{l}$ the finite leximax criterion. Let $\left\{a_{1}, \ldots a_{k}\right\}$ and $\left\{b_{1}, \ldots b_{r}\right\}$ be two subsets of $X$, whose elements are labelled from best to worst (with respect to $R$ ). We consider $A \succeq_{l} B$ when

i) there exists $i \leq k$ such that $a_{i} P b_{i}$ and $a_{j} I b_{j}$, for all $j<i$, or

ii) $a_{i} I b_{i}$, for all $i \leq r$ and $k \geq r$.

Next, two preference relations on $2^{X}$ are considered. We denote by $\succeq_{L}^{*}$ the leximax criterion defined in Ballester and De Miguel (2003): For any pair of sets $A, B \subseteq X, A \succeq_{L}^{*} B$ if and only if for all $B_{f} \in \mathcal{F}(B)$ there exists $A_{f} \in \mathcal{F}(A)$ such that $A_{f} \succeq_{l} B_{f}$.

We denote by $\succeq_{L}$ the leximax criterion of Arlegi et al. (2005), who use a

3 There are some slight differences between the domains considered in each article. Basically, the definition of Ballester and De Miguel (2003) is more general in that it does not require the existence of a utility representation. In this article we have adopted a common formal framework for the sake of comparability. 
more procedural definition, the presentation of which requires some additional notation. Let $u: X \rightarrow[0,1]$ be such that for all $x, y \in X, u(x) \geq u(y)$ iff $x R y$, that is, $u$ is a utility function for the preference relation over the alternatives.

Let $A \subseteq X$; we denote by $u(A)$ the set $\{u(a): a \in A\}$. Since $u(A)$ is a bounded set, there exists $\sup u(A)$. If there exists $a \in A$ such that $u(a)=\sup u(A)$, this $a$ will be denoted by $a_{1}$. In this case, we consider the bounded set $u\left(A \backslash\left\{a_{1}\right\}\right)$, then there exists $\sup u\left(A \backslash\left\{a_{1}\right\}\right)$; again, if there exists $a \in A \backslash\left\{a_{1}\right\}$ such that $u(a)=\sup u\left(A \backslash\left\{a_{1}\right\}\right)$, this $a$ will be denoted by $a_{2}$, and so on.

Given a (possibly infinite) set $X$, the leximax ordering on $2^{X}, \succeq_{L}$, is defined as follows: First, for all $A \neq \emptyset, A \succ_{L} \emptyset \sim_{L} \emptyset$. Second, let $A, B \subseteq X, A, B \neq \emptyset$, there are three possibilities:

1) $a_{1}$ exists but not $b_{1}$.

1.1 If $a_{1} P x$, for all $x \in B$, then $A \succ_{L} B$.

1.2 If there exists $x \in B$ such that $x P a_{1}$, then $B \succ_{L} A$.

2) Neither $a_{1}$ nor $b_{1}$ exists.

2.1 If $\sup u(A)>\sup u(B)$, then $A \succ_{L} B$.

2.2 If $\sup u(B)>\sup u(A)$, then $B \succ_{L} A$.

2.3 If $\sup u(A)=\sup u(B)$ then $A \sim_{L} B$.

3) Both $a_{1}$ and $b_{1}$ exist.

3.1 If $a_{1} P b_{1}$, then $A \succ_{L} B$.

3.2 If $b_{1} P a_{1}$, then $B \succ_{L} A$.

3.3 If $a_{1} I b_{1}$, it could be that:

3.3.a $A=\left\{a_{1}\right\}$ and $B=\left\{b_{1}\right\}$, in which case $A \sim_{L} B$.

3.3.b $A=\left\{a_{1}\right\}$ and $B \neq\left\{b_{1}\right\}$. In which case $B \succ_{L} A$. Analogously, if $A \neq\left\{a_{1}\right\}$ and $B=\left\{b_{1}\right\}$, then $A \succ_{L} B$.

3.3.c $A \neq\left\{a_{1}\right\}$ and $B \neq\left\{b_{1}\right\}$. In this case we consider the sets $A \backslash\left\{a_{1}\right\}$ and $B \backslash\left\{b_{1}\right\}$. If $A \backslash\left\{a_{1}\right\}$ and $B \backslash\left\{b_{1}\right\}$ are in one of the cases (from 1 to 3.3.b) above, then we conclude $A \succeq_{L} B$ iff $A \backslash\left\{a_{1}\right\} \succeq_{L} B \backslash\left\{b_{1}\right\}$. Otherwise, we repeat the procedure as often as required to find $k \in \mathbb{N}$ such that $a_{i} I b_{i}, i=1, \ldots, k$, and $A \backslash\left\{a_{1}, \ldots, a_{k}\right\}, B \backslash\left\{b_{1}, \ldots, b_{k}\right\}$ are in one of the cases 1 to 3.3.b, and conclude that $A \succeq_{L} B$ if $A \backslash\left\{a_{1}, \ldots, a_{k}\right\} \succeq_{L} B \backslash\left\{b_{1}, \ldots, b_{k}\right\}$. 
The only case in which this algorithm does not come to an end is when, for all $k \in \mathbb{N}$, there exist $a_{k} \in A, b_{k} \in B$ such that, $a_{k} I b_{k}$. In that case, we say that $A \sim_{L} B$

\section{The main result}

Theorem 1 The two preference relations defined on $2^{X}, \succeq_{L}$ and $\succeq_{L}^{*}$, are the same.

\section{Proof of Theorem 1}

Let $A, B \subseteq X$ such that $A \succeq_{L} B$. We prove that $A \succ_{L} B$ implies $A \succ_{L}^{*} B$ and that $A \sim_{L} B$ implies $A \sim_{L}^{*} B$.

First, note that, whenever the empty set is involved in the comparison, $A \succ_{L}^{*}$ $\emptyset \sim_{L}^{*} \emptyset$. Thus, we will concentrate on the remaining comparisons. For notational convenience throughout this proof, we assume that for the comparison between the sets $A$ and $B$, the first step of the procedure (whether $a_{1}$ and $b_{1}$ exist or not) is the decisive one. Note that if there exists a finite number of pairs of initial elements which are indifferent two by two, and subsequently this situation is not repeated, then the reasoning is not affected by removing the initial pairwise equal elements.

We start by supposing that $A \sim_{L} B$. There are two possible cases:

Case 2.3 Neither $a_{1}$ nor $b_{1}$ exists, and $\sup u(A)=\sup u(B)$. Then $\sup u(B)>$ $\sup u\left(B_{f}\right)$ for all $B_{f} \in \mathcal{F}(B)$, therefore there exists $a \in A$ such that $a P b$, for all $b \in B_{f}$ and for all $B_{f} \in \mathcal{F}(B)$. Thus, $\{a\} \succ_{l} B_{f}$ and $A \succeq_{L}^{*} B$. Analogously it is proved that $B \succeq_{L}^{*} A$. Consequently $A \sim_{L}^{*} B$.

Case 3.3 Both $a_{1}$ and $b_{1}$ exist and $a_{1} I b_{1}$. If $A=\left\{a_{1}\right\}$ and $B=\left\{b_{1}\right\}$, then $A \sim_{L}^{*} B$. Consider now the case where for all $k \in \mathbb{N}$, there exists $a_{k} \in A$, $b_{k} \in B$ and $a_{k} I b_{k}$. Then, for any $B_{f} \in \mathcal{F}(B)$, we only need to consider the set containing the $\left|B_{f}\right|$ top elements of $A$ in order to obtain $A \succsim_{L}^{*} B$. Similarly $B \succsim_{L}^{*} A$, leading to $A \sim_{L}^{*} B$.

Suppose now that $A \succ_{L} B$. If any of the cases 1.1, 1.2, 2.1, 3.1 is at stake, the following condition holds: there exists an element $a \in A$ such that $a P x$ for all $x \in B$. In this case, obviously, $\{a\} \succ_{l} B_{f}$ for any finite subset of $B$, thus implying $A \succ_{L}^{*} B$.

Finally, if case 3.3 is the relevant one, $A \neq\left\{a_{1}\right\}$ whereas $B=\left\{b_{1}\right\}$ with $b_{1} I a_{1}$. Consider then any element $a \in A \backslash\left\{a_{1}\right\}$. Obviously, $\left\{a_{1}, a\right\} \succ_{l} B$, where $B$ is 
the only finite subset of $B$, implying $A \succ_{L}^{*} B$.

\section{The axioms}

As pointed out earlier, the axiomatic characterizations of the leximax in Ballester and De Miguel (2003), and in Arlegi et al. (2005) are different. Next, we present the axioms used in each article and briefly comment on the logical relationships among them. For the sake of fluency, we assume throughout that $R$ is a linear ordering. While this affects the wording of some of the axioms, the logical relationships between them remain unchanged up to the corresponding restatements of them. ${ }^{4}$

Ballester and De Miguel (2003) characterize their extension of the leximax by means of the following axioms:

\section{Independence (In)}

For all $A, B \in 2^{X}$, for all $x \in X \backslash(A \cup B): A \succeq B \Longleftrightarrow A \cup\{x\} \succeq B \cup\{x\}$.

\section{Robustness (Rb)}

For all $A, B, C \in 2^{X}$ such that $x P y$ for all $x \in A \cup B$ and all $y \in C: A \succ$ $B \Longrightarrow A \succ B \cup C$

\section{No-Choice Situations (NCS)}

For all $A \in 2^{X}: A \neq \emptyset$ implies $A \succ \emptyset$

\section{Existence of a Significant Element (SE)}

For all $A, B \in 2^{X}$, with $A \succ B$, there exists $a \in A \backslash B$ such that the set $\{b \in B: b R a\}$ is finite.

Independence and Robustness are used by Bossert et al. (1994) for the characterization of the leximax criterion in finite environments. These axioms are also preserved also in Arlegi et al. (2005) for the characterization. ${ }^{5}$ Furthermore, Arlegi et al. (2005) take (NCS) as a general assumption, and, instead of (SE), propose the two following additional axioms:

$\overline{4}$ We think that the discussion of both the motivation and technical details of the axioms is beyond the scope of this note. We refer the interested reader to the original articles.

5 Axiom (Rb) as presented by Arlegi et al. (2005) is slightly stronger, but their proof also runs with the weaker version proposed by Ballester and De Miguel (2003). 


\section{Dominance}

Let $A, B \subseteq X$, if, for all $b \in B$, there exists $a \in A$ such that $a P b$, then $A \succeq B$.

\section{Indifference}

Let $A, B \subseteq X$. If there exist two one-to-one functions $\alpha: \mathbb{N} \rightarrow A$ and $\beta: \mathbb{N} \rightarrow$ $B$ such that for all $k \in \mathbb{N}, \alpha(k)=\beta(k), \alpha(k) R a$ for all $a \in A \backslash\{\alpha(1), \ldots, \alpha(k-$ $1)\}$ and $\beta(k) R b$ for all $\beta \in B \backslash\{\beta(1), \ldots, \beta(k-1)\}$, then $A \sim B$.

Therefore, the difference between the two characterizations is that Arlegi et al. (2005) use Dominance and Indifference instead of Ballester and De Miguel's (2003) (SE) axiom. Concerning the logical relationship between these axioms, it can be proved that (SE) implies Indifference but not Dominance, and also that neither Dominance nor Indifference separately implies (SE). Furthermore, it can be proved that Dominance and Indifference together do not imply (SE) (obviously, in the absence of the remaining axioms). Since this is just a brief research note, we do not present the proofs. They are, however, available upon request.

\section{Conclusions}

In this work we have proved that the two so far existing extensions of the leximax rule to infinite environments are equivalent. Before this result was proved we had two possible ways of applying the leximax in infinite environments, each with advantages that were lacking the other: The leximax extension by Ballester and De Miguel (2003) is more synthetic and "compact". We think it is mathematically more elegant and useful, for example, as a tool for the investigation of further results. On the other hand, the proposal by Arlegi et al. (2005) is more extensive and "procedural", and perhaps easier to apply as a quick means to decide how to rank any pair of given sets: According to the criterion by Ballester and De Miguel (2003), in order to declare a set $A$ as preferred to another set $B$ one has to be sure that for every finite set in $B$ there exists another finite set in $A$ that is better in terms of the (finite) leximax, which might not be immediate to check in many instances.

The equivalence result we have proved means that it is possible to use whichever extension of the leximax best meets one's requirements, in the certainty that this will not affect the ranking of the set, or potential further results.

\section{Acknowledgements}

We thank an anonymous referee for his/her comments on a previous version of 
this work. This research has been supported by Spanish Ministry of Education grants SEC2003-08105 (a), BEC2002-3780 (c) from DGICYT, and SEJ200501481/ECON, FEDER and CREA (b).

\section{References}

[1] Arlegi, R., Besada, M., Nieto, J., Vázquez, C., 2005. Freedom of choice: the leximax criterion in the infinite case. Mathematical Social Sciences 49, $1-15$.

[2] Ballester, M., De Miguel, J.R., 2003. Extending an order to the power set: the leximax criterion. Social Choice and Welfare 21, 63-71.

[3] Barberà, S., Bossert, W., Pattanaik, P., 2004. Ranking Sets of Objects. In: Barberà, S., Hammond, P., Seidl, C., (Eds.), Handbook of Utility Theory, Vol. 2. Kluwer Academic Publishers, pp. 893-979.

[4] Bossert, W., Pattanaik, P., Xu, Y., 1994. Ranking Opportunity Sets: An Axiomatic Approach. Journal of Economic Theory 63, 326-345.

[5] Pattanaik, P., Xu, Y., 1990. On Ranking Opportunity Sets in Terms of Freedom of Choice. Recherches Economiques de Louvain 56, 383-390. 